\title{
Suppression of Instability on Sensing Signal of Optical Pulse Correlation Measurement in Remote Fiber Sensing
}

\author{
Hirokazu Kobayashi, ${ }^{1}$ Toshimasa Tsuzuki, ${ }^{1}$ Toshitake Onishi, ${ }^{1}$ Yuhei Masaoka, ${ }^{1}$ \\ Xunjian $\mathrm{Xu}^{2}$ and Koji Nonaka ${ }^{1}$ \\ ${ }^{1}$ Department of Electronic and Photonic Systems Engineering, Kochi University of Technology, Kochi 782-8502, Japan \\ ${ }^{2}$ Key Laboratory, State Grid Corporation of China, Beijing 100031, China
}

Correspondence should be addressed to Hirokazu Kobayashi, hirokazu.kobayashi@gmail.com

Received 20 July 2012; Revised 7 November 2012; Accepted 8 November 2012

Academic Editor: Hao Zhang

Copyright (C) 2012 Hirokazu Kobayashi et al. This is an open access article distributed under the Creative Commons Attribution License, which permits unrestricted use, distribution, and reproduction in any medium, provided the original work is properly cited.

\begin{abstract}
Optical fiber sensing has the potential to overcome weak points of traditional electric sensors. Many types of optical fiber sensors have been proposed according to the modulation parameter of incident light. We have proposed an optical pulse correlation sensing system that focuses on the time drift values of the propagating optical pulses to monitor the temperature- or straininduced extension along the optical fiber in the sensing region. In this study, we consider the instability in the optical pulse correlation sensing system applied to remote monitoring over a kilometer-long distance. We introduce a method to stabilize the instability of the pulse correlation signal resulting from the time drift fluctuation along a transmission line. By using this method, we can purify the response and improve the accuracy of signals at the focused sensing regions. We also experimentally demonstrate remote temperature monitoring over a $30 \mathrm{~km}$-long distance using a remote reference technique, and we estimate the resolution and the measurable span of the temperature variation as $(1.1 / L)^{\circ} \mathrm{C}$ and $(5.9 \times 10 / L)^{\circ} \mathrm{C}$, respectively, where $L$ is the length of the fiber in the sensing region.
\end{abstract}

\section{Introduction}

In recent years, optical fiber sensors have attracted growing interest for a variety of reasons, including their corrosion resistance, electromagnetic immunity, long lifetime components, high sensitivity, and multiplexing capability [1-6]. This sensor impresses information onto the light beam in response to environmental parameters such as temperature and strain. The information could be impressed in terms of intensity, phase, wavelength, and time. In the course of their study, many types of optical fiber sensors have been proposed, such as in-fiber Bragg grating (FBG) sensors [7], low-coherence interferometric sensors [8], Fabry-Pérot sensors $[9,10]$, Brillouin scattering distributed sensors [11], and Raman scattering distributed sensors [12]. It has been expected that the optical fiber sensors would be used for the monitoring of complicated composite structures because they are capable of measuring strain and temperature over several tens of kilometers by accessing only one end of an optical fiber [13, 14]. Feasibility studies have been performed by using actual structures, for example, bridges [15-17], dams [18], marine vehicles [19], and aircrafts [20,21].

In our laboratory, we have proposed and developed an optical pulse correlation sensing system in which the sensing signals are the time drift values of the optical pulses caused by the temperature- or strain-induced extension along the optical fiber in the sensing region [22-26]. This sensing system is not only simple and handy, but also highly accurate for temperature or strain measurement [24]. It is notable that our fiber sensor can work as a line sensor, that is, the time drift caused by the expansion of the optical fiber is integrated along the sensing region, and the sensing signal is proportional to the integrated time drift. Thus, we can select the sensitivity by changing the fiber length in the sensing region.

Herein, we consider the optical pulse correlation sensing system applied to remote monitoring over a kilometer-long distance. For this purpose, a kilometer-long optical fiber is 
used for the transmission line connecting the monitoring center and the sensing region. In this case, however, a minute temperature or strain fluctuation around the transmission line is integrated along the long optical fiber and induces some instability in the sensing signals, for example, power, polarization, and time drift fluctuations.

In this paper, we focus on the time drift fluctuations and estimate its effect on signal drift. Moreover, we introduce a method to suppress the fluctuation by using the region separation technique with a partial reflector [27]. We also experimentally demonstrate remote temperature sensing over a $30 \mathrm{~km}$-long transmission line and estimate the resolution and the measurable span of the temperature variation.

The remainder of this paper is organized as follows. In Section 2, we introduce the principle of the optical pulse correlation sensing system. In Section 3, we experimentally confirm the time drift fluctuation of the sensing signal caused by the long transmission line. Then, we propose a modified experimental setup that can stabilize the sensing signal against the time drift fluctuation in Section 4. In this setup, the fluctuation is suppressed by use of a remote reference pulse. Moreover, we demonstrate remote temperature monitoring over a $30 \mathrm{~km}$ distance. A conclusion is presented in Section 5.

\section{Principle of the Optical Pulse Correlation Sensing System}

2.1. Conventional Sensing System Using Optical Pulse Correlation Measurement. A schematic of our conventional system is shown in Figure 1. First, we generate two optical pulses, a reference pulse $(R)$ and a monitoring pulse $(M)$, at the monitoring center. The monitoring pulse passes through a transmission line and then enters a sensing region. After that, the monitoring pulse is reflected back to the monitoring center and enters a pulse correlation measurement unit. In contrast, the reference pulse directly enters the pulse correlation measurement unit. If the temperature or strain in the sensing region is changed, the optical path in the measuring fiber produces a variation of relative time drift $\tau$ between the reference and monitoring pulses. From the variation of the time drift value measured by the pulse correlation measurement, we can estimate the temperature or strain variation.

2.2. Pulse Correlation Measurement Unit. A schematic of the pulse correlation measurement unit is shown in Figure 2. In this system, a reference pulse with $45^{\circ}$ polarization is split into two orthogonally-polarized pulses, $R_{1}$ (horizontal polarization) and $R_{2}$ (vertical polarization), with a fixed timing separation $\tau_{0}$ induced by a birefringence crystal (pulse doubler). The monitoring pulse with $45^{\circ}$ polarization is combined with doubled reference pulses and separated into two channels by using a polarized beam splitter. Thus, one channel has a backward reference pulse $R_{1}$ and a monitoring pulse with a time drift $\tau_{0}-\tau$, while the other channel has a forward reference pulse $R_{2}$ and a monitoring pulse with a time drift $\tau$.

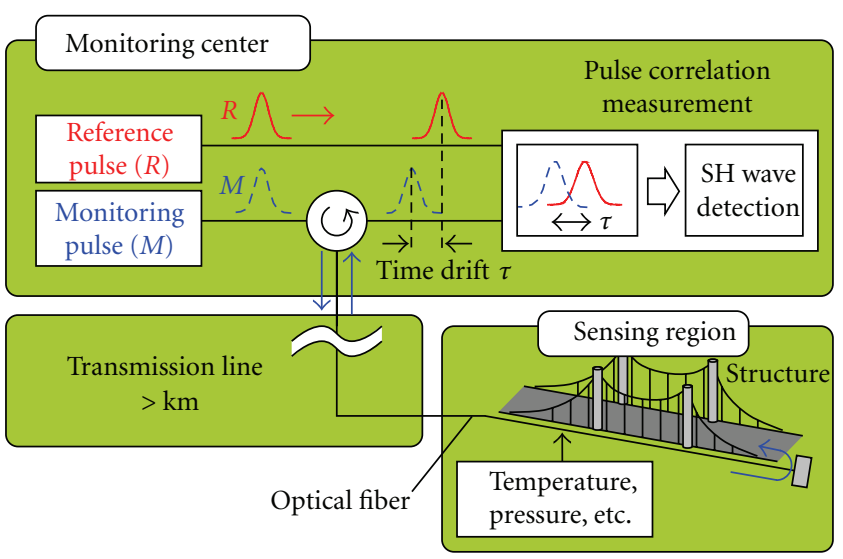

FIGURE 1: Schematic of remote fiber sensing using optical pulse correlation measurement.

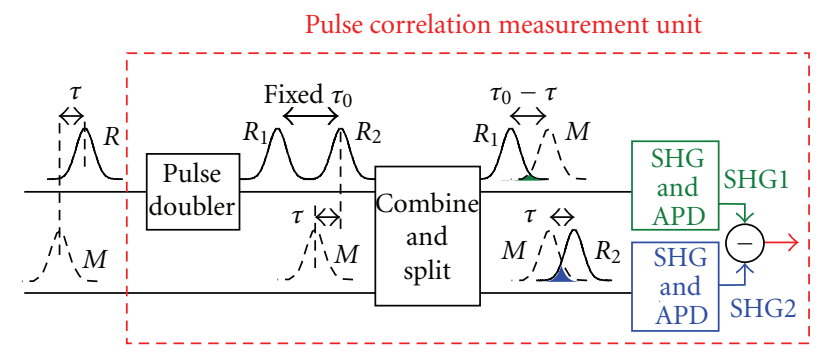

Figure 2: Schematic of pulse correlation measurement unit. $R$ : reference pulse, $M$ : monitoring pulse, SHG: second harmonic generation, and APD: avalanche photodiode.

In each channel, the second harmonic generation (SHG) signals are observed by two avalanche photodiodes (APDs). The SHG output is maximized when the monitoring pulse completely overlaps with the reference pulse and its full width at half maximum is related to the pulse width $\delta t$ of the incident pulsed laser light. The green dashed line and the blue dotted line in Figure 3 show the theoretical plots of the typical relationship between the time drift $\tau$ and SHG outputs. To obtain a wide-range linear response with respect to the time drift value, we consider the differential signal between two SHG outputs (see the red solid line in Figure 3). The important part of the differential signal curve is highlighted inside a dashed oval in Figure 3. In this region, the output relationship shows good linearity with respect to the time drift value. In what follows, we estimate the time drift value from the differential signal by using this linear relationship.

2.3. The Variation of the Time Drift Value Owing to Temperature or Strain Changes. The variation of the time drift value $\Delta \tau$ within the fiber length $L$ can be expressed in terms of the temperature change $\Delta T$ and the strain change $\Delta F$ :

$$
\begin{gathered}
\Delta \tau=L \cdot f, \\
f \equiv C_{T} \Delta T+C_{F} \Delta F,
\end{gathered}
$$




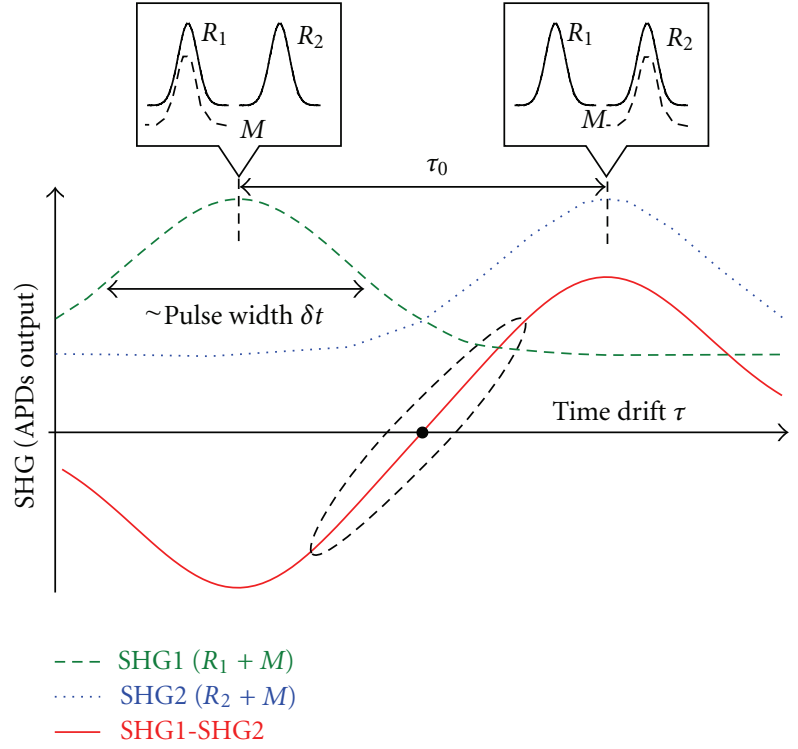

Figure 3: Theoretical plots of SHG signals (green dashed line and blue dotted line) and their differential signal (red solid line) with respect to the relative time drift $\tau$.

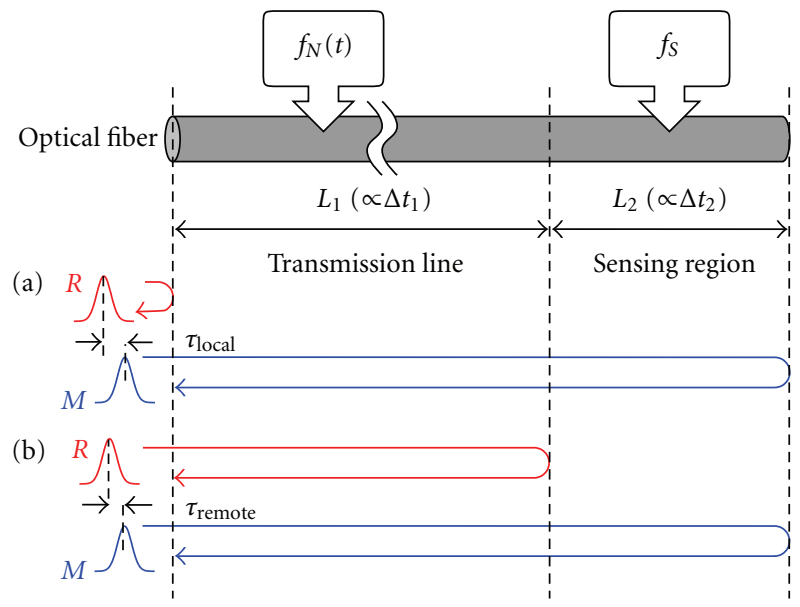

FIgure 4: The time drift value in the optical pulse correlation sensing system when we use (a) a local reference pulse and (b) a remote reference pulse.

where $f$ is the variation of the time drift value per unit length of the optical fiber, and $C_{T}$ and $C_{F}$ are the temperature sensitivity and the strain sensitivity, respectively. For standard commercial single mode fiber at wavelength $1550 \mathrm{~nm}$ with a diameter of $2 \mathrm{~mm}$, the typical values are $C_{T}=0.17 \mathrm{ps} /\left(\mathrm{m} \cdot{ }^{\circ} \mathrm{C}\right)[26]$ and $C_{F}=2.7 \mathrm{ps} /(\mathrm{m} \cdot \mathrm{N})[28]$.

Now, we consider the measurement of the temperature or strain changes around the sensing region with a length of $L_{2}$ over the transmission line with a length of $L_{1}$ (see Figure 4$). f_{S}$ and $f_{N}$ are variations of the time drift value per unit length along the sensing region and the transmission line, respectively. We assume that $f_{N}$ is dependent on the time $t$, that is, there is the temporal fluctuation of temperature or strain around the transmission line.
The conventional situation of the optical pulse correlation sensing system is shown in Figure 4(a), where the monitoring pulse is sent to the sensing region through the transmission line while the reference pulse is maintained in the monitoring center (we call it the "local" reference pulse). We approximate the temporal variation of $f_{N}(t)$ caused by the propagation time in the transmission line $\left(\Delta t_{1}\right)$ and in the sensing region $\left(\Delta t_{2}\right)$ by a first-order Taylor expansion. The variation of the time drift value $\Delta \tau_{\text {local }}$ is calculated as

$$
\begin{aligned}
\Delta \tau_{\text {local }} & \simeq 2\left[L_{2} f_{S}+L_{1}\left\{f_{N}(t)+\frac{\mathrm{d} f_{N}}{\mathrm{~d} t}\left(\Delta t_{1}+\Delta t_{2}\right)\right\}\right] \\
& \simeq 2\left\{L_{2} f_{S}+L_{1} f_{N}(t)\right\},
\end{aligned}
$$

where we assume $f_{N}(t) \gg\left(\mathrm{d} f_{N} / \mathrm{d} t\right) \Delta t_{i}(i=1,2)$ and $\Delta t_{i} \equiv n_{g} L_{i} / c(i=1,2)$ with the speed of light $c$ and the refractive index for the group velocity $n_{g}$. The first term in (3) corresponds to the sensing signal, and the second term in (3) shows the effect of the time drift fluctuation caused by the transmission line. If the second term is sufficiently small compared to the first term, we can estimate the temperature or strain changes within the sensing region from the time drift value $\Delta \tau$. In case of the long transmission line, however, $L_{1} f_{N}(t)$ could become large enough to induce the appreciable time drift fluctuation.

To suppress this fluctuation, we can utilize a "remote reference pulse," that is, an optical pulse reflected by a partial reflector just before the sensing region (see Figure 4(b)). This idea is inspired by region separation techniques used to realize multiple region sensing [27]. In this case, the variation of the time drift value $\Delta \tau$ is calculated as

$$
\Delta \tau_{\text {remote }} \simeq 2\left[L_{2} f_{S}+L_{1} \frac{\mathrm{d} f_{N}}{\mathrm{~d} t} \Delta t_{2}\right] .
$$

By using a remote reference pulse, the main factor $L_{1} f_{N}(t)$ of the time drift fluctuation caused by the transmission line is canceled out because both the reference and monitoring pulses are passing through the transmission line. The second term in (4) shows the effect of temporal variation fluctuation caused by the difference of the propagation time between the reference and monitoring pulse. If the second term in (4) is sufficiently small compared to the first term, $\Delta \tau_{\text {remote }}$ can be stabilized against the time drift fluctuation by using remote reference pulses.

From (2), our sensing system has the cross sensitivity between temperature and strain changes. The simplest way of discriminating strain and temperature changes is to use two optical fibers where the first one is not fixed at the measuring object, that is, isolated from strain and experiences only temperature changes, and the second one is fixed at the measuring object, that is, affected by both strain and temperature. Assuming that the two sensing fibers are at the same temperature, the variation of the time drift value from the first sensor can be used to derive a temperaturecorrected strain value from the second one. In what follows, the optical fiber is not fixed at the measuring object, and we monitor only temperature changes. 


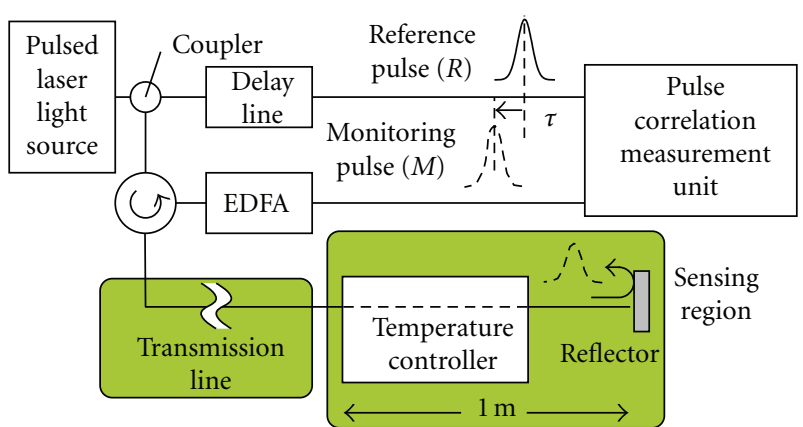

Figure 5: Experimental setup for remote fiber sensing using pulse correlation measurement.

\section{Experimental Observation of Time Drift Fluctuation in the Long Transmission Line}

Figure 5 shows the experimental setup for remote fiber sensing using pulse correlation measurement. We use optical pulses with a repetition frequency of $1.4794 \mathrm{GHz}$, a center wavelength of $1550 \mathrm{~nm}$, and a pulse width of around $10 \mathrm{ps}$ as light source. First, the input pulses are split into two pulses, reference pulse and monitoring pulse, by a coupler. The monitoring pulse passes through an optical circulator and a transmission line and then enters a sensing region. After that, the monitoring pulse is reflected back to the circulator and then enters a pulse correlation measurement unit subsequent to amplification by an erbium-doped fiber amplifier (EDFA), which compensates for propagation loss in the transmission line. In contrast, the reference pulse directly enters the pulse correlation measurement unit through a tunable delay line that is fixed at a point to allow a partial overlap between the reference and monitoring pulses. The delay of the tunable delay line device (OZ Optics ODL300) can be adjusted with 0.005 -ps increments between 0 and 350 ps. In the correlation measurement unit, the fixed timing separation $\tau_{0}$ is $20 \mathrm{ps}$. The outputs of two APDs are connected to a 12-bit analog to digital converter (ADC) to obtain the SHG output power as the voltage value. Finally, the output differential signal between two SHG outputs is calculated and stored in a personal computer.

Figure 6 shows experimentally obtained SHG outputs and their differential signal with respect to the time position of the tunable delay line. The linear region with a width of about 10 ps can be used for the estimation of the time drift value. As described in Section 2.3, the temperature sensitivity is $0.17 \mathrm{ps} /\left(\mathrm{m} \cdot{ }^{\circ} \mathrm{C}\right)$. Thus, the measurable span of the temperature variation is calculated as $\left(5.9 \times 10 / L_{2}\right)^{\circ} \mathrm{C}$, where $L_{2}$ is the length of the fiber in the sensing region. This indicates that the measurable span becomes smaller when we use a longer optical fiber.

Next, we stabilized the temperature of a $1 \mathrm{~m}$ long sensing region at room temperature $\left(\sim 27^{\circ} \mathrm{C}\right)$ by using a feedback loop of the temperature controller and continuously monitoring the time drift value to observe the time drift fluctuation at a fixed temperature in the sensing region. The experimental results are shown in Figure 7.

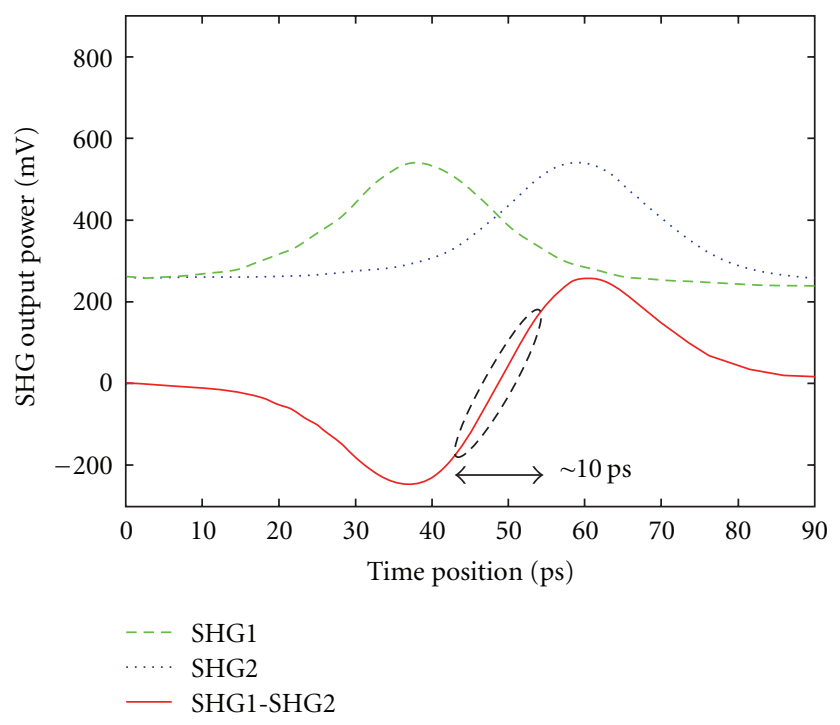

FIGURE 6: Experimentally obtained SHG outputs (green dashed line and blue dotted line) and their differential signal (red solid line).

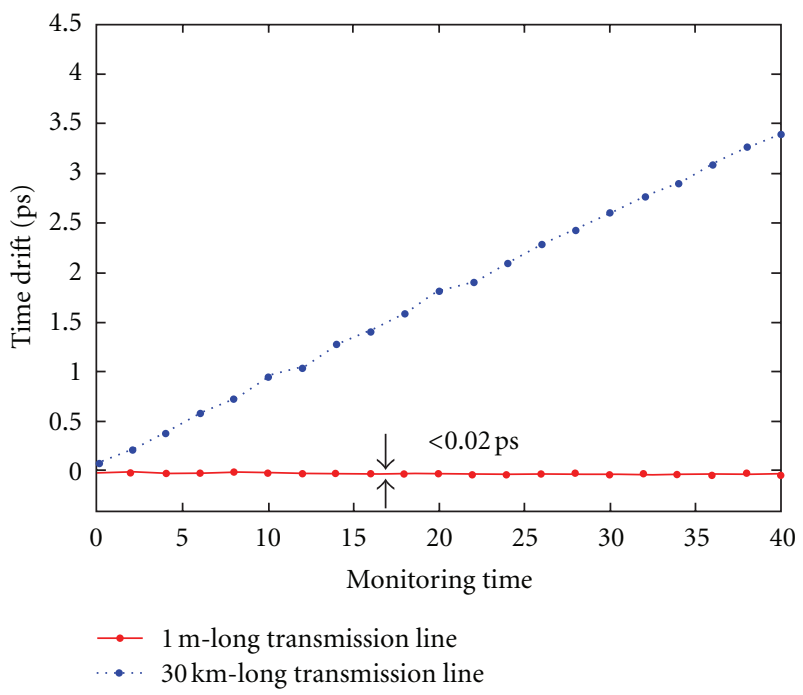

FIGURE 7: Time drift fluctuation in remote monitoring of the $1 \mathrm{~m}$ long sensing region at the fixed temperature $27.0 \pm 0.1^{\circ} \mathrm{C}$. The solid red line and the dotted blue line show the fluctuation of the time drift value with monitoring time through short $(\sim 1 \mathrm{~m})$ and long $(\sim 30 \mathrm{~km})$ transmission lines, respectively.

For the short transmission line $(\sim 1 \mathrm{~m})$, the time drift value remains almost constant with respect to the monitoring time (see the solid red line in Figure 7). Actually, the time drift fluctuation is less than $0.02 \mathrm{ps}$, which is within the 12-bit resolution of the ADC. From this value and the sensitivity coefficient, the temperature resolution of this system is calculated as $\left(1.2 \times 10^{-1} / L_{2}\right)^{\circ} \mathrm{C}$. We can adjust the temperature resolution to satisfy the corresponding application requirements by changing the fiber length $L_{2}$.

For the long transmission line $(\sim 30 \mathrm{~km})$, however, even in the constant-temperature sensing region, the measured time drift value shifts gradually with monitoring time at 


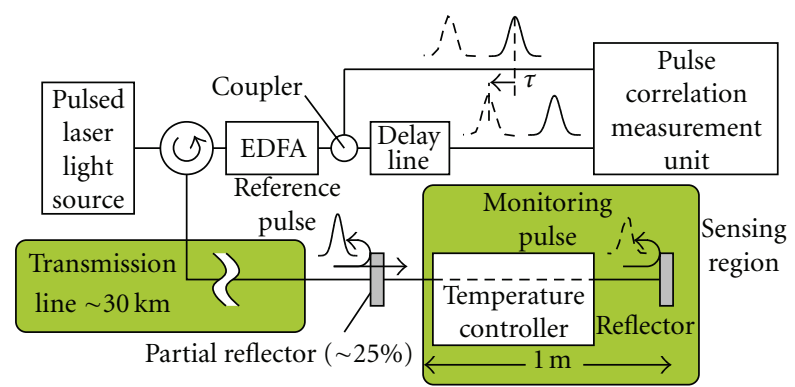

Figure 8: Experimental setup for remote optical fiber sensing using a remote reference pulse.

a speed of 0.09 psper second (see the dotted blue line in Figure 7). Because the measurable span is about $10 \mathrm{ps}$, only a few minutes of the monitoring time is required to break the linear response with respect to the time drift value.

From (3), we can calculate the speed of the temperature variation around the transmission line as:

$$
\begin{gathered}
L_{1} \frac{\mathrm{d} f_{N}}{\mathrm{~d} t}=L_{1} C_{T} \frac{\mathrm{d} \Delta T}{\mathrm{~d} t}=0.09 \mathrm{ps} / \mathrm{s}, \\
\therefore \frac{\mathrm{d} \Delta T}{\mathrm{~d} t}=1.8 \times 10^{-5}{ }^{\circ} \mathrm{C} / \mathrm{s} .
\end{gathered}
$$

This minute variation is caused by the ambient temperature and the warming by optical pulses. It is integrated along the long transmission line and induces an appreciable time drift fluctuation. To realize remote temperature monitoring, we need to suppress the large time drift fluctuation in the long transmission line.

\section{A Pulse Correlation Sensing System Using a Remote Reference Pulse}

4.1. Experimental Setup. From (5), we can calculate the second term in (4) as $4.4 \times 10^{-22} \mathrm{~s}$. This result is sufficiently small compared to the first term in (4). Thus, we can suppress the time drift fluctuation by utilizing a remote reference pulse.

The experimental setup with the remote reference pulse is shown in Figure 8. In this setup, the reference pulse is reflected by a partial reflector with a reflectivity of about $25 \%$, and the monitoring pulses are reflected at the end of the same optical fiber. After amplification by the EDFA, the reflected pulses including the reference and monitoring pulses are split into two pulses. The tunable delay line is set up to allow partial overlap between the reference pulse in one arm and the monitoring pulse in the other arm.

4.2. Experimental Results. The continuous monitoring of the time drift value is shown in Figure 9 when the temperature in the $1 \mathrm{~m}$ long sensing region is fixed at room temperature. We can easily confirm that the time drift value is sufficiently stabilized in monitoring time by using a remote reference pulse. The time drift fluctuation is below 0.2 ps even in an hour over the $30 \mathrm{~km}$-long transmission line. This indicates

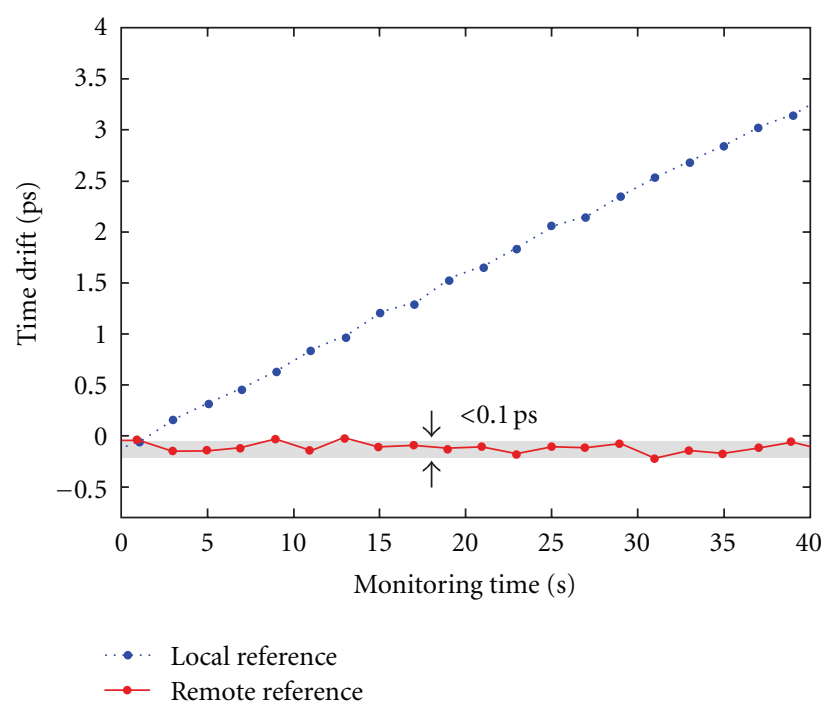

FIGURE 9: Time drift fluctuation in remote monitoring over a $30 \mathrm{~km}$ distance of the $1 \mathrm{~m}$ long sensing region at the fixed temperature $27.0 \pm 0.1^{\circ} \mathrm{C}$ by using the local reference pulse (dotted blue line) and the remote reference pulse (red solid line).

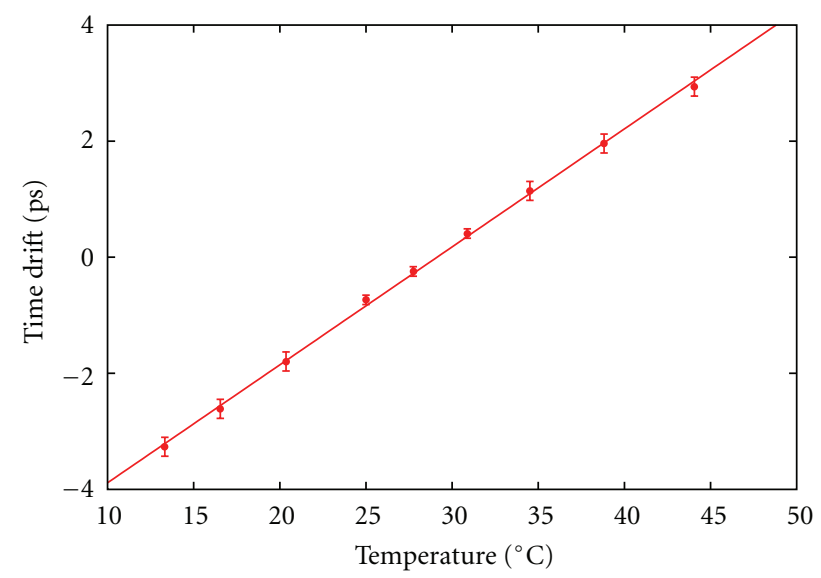

Figure 10: Remote temperature monitoring at a $1 \mathrm{~m}$ long sensing region over a $30 \mathrm{~km}$-long transmission line by utilizing a remote reference.

that the temperature resolution is $\left(1.1 / L_{2}\right)^{\circ} \mathrm{C}$ and the measurable span of the temperature variation is $\left(5.9 \times 10 / L_{2}\right)^{\circ} \mathrm{C}$.

Next, we demonstrate remote temperature monitoring. The time drift value is continuously monitored with the changing surrounding temperature of the optical fiber in the sensing region. Figure 10 shows the change of the measured time drift value using the remote reference with respect to the temperature around the $1 \mathrm{~m}$ long monitoring fiber. The good linearity between the measured time drift value and the temperature of the sensing region can be clearly observed by suppressing the effect of long-distance instability. From the result, the temperature sensitivity of the $1 \mathrm{~m}$ long monitoring fiber is found to be about $0.18 \mathrm{ps} /{ }^{\circ} \mathrm{C}$, which is almost equal to the value in [26]. The temperature resolution estimated from the error bar in Figure 10 is less than $1.6^{\circ} \mathrm{C}$. 


\section{Conclusions}

In this paper, we introduced an optical fiber sensing system for long-distance remote monitoring using pulse correlation measurement. We estimated the effect of the time drift fluctuation from the long transmission lines and proposed a stabilizing method using a remote reference pulse against the time drift fluctuation caused by the long transmission line. Moreover, we demonstrated remote temperature monitoring over the $30 \mathrm{~km}$-long transmission line by utilizing a remote reference pulse and obtained good linearity of the time drift value with respect to the temperature around the sensing region. The resolution and the measurable span of the temperature variation are $\left(1.1 / L_{2}\right)^{\circ} \mathrm{C}$ and $\left(5.9 \times 10 / L_{2}\right)^{\circ} \mathrm{C}$, respectively. We can select the resolution and the measurable span by changing the length of the optical fiber in the sensing region. This system can be used for remote temperature monitoring of complicated structures, such as bridges, oil tanks, power transformers, and pipelines.

\section{Acknowledgments}

This work was partially supported by Y2009-2010 Practical Application Research no. 1513 of Japan Science and Technology Agency (JST) and the Japan Society for the Promotion of Science (JSPS) no. 18360180 and no. 22657062.

\section{References}

[1] E. Udd and W. B. Spillman Jr., Eds., Fiber Optic Sensors: An Introduction for Engineers and Scientists, John Wiley \& Sons, New York, NY, USA, 1991.

[2] J. Dakin and B. Culshaw, Optical Fiber Sensors: Principles and Components, vol. 1, Artech House, Boston, Mass, USA, 1988.

[3] K. T. V. Grattan and B. T. Meggitt, Eds., Optical Fiber Sensor Technology, Volume 2: Devices and Technology, Chapman \& Hall, London, 1997.

[4] T. G. Giallorenzi, J. A. Bucaro, A. Dandridge et al., "Optical fiber sensor technology," IEEE Journal of Quantum Electronics, vol. 18, no. 4, pp. 626-665, 1982.

[5] F. T. S. Yu and S. Yin, Eds., Fiber Optic Sensor, Marcel Dekker, New York, NY, USA, 2002.

[6] L. Thévenaz, "Review and progress in distributed fiber sensing," in Proceedings of the Optical Fiber Sensors, Cancuún, Mexico, 2006, Paper ThC1.

[7] Y. J. Rao, "In-fibre Bragg grating sensors," Measurement Science and Technology, vol. 8, no. 4, p. 355, 1997.

[8] Y. J. Rao and D. A. Jackson, "Recent progress in fibre optic lowcoherence interferometry," Measurement Science and Technology, vol. 7, no. 7, p. 981, 1996.

[9] A. D. Kersey, D. A. Jackson, and M. Corke, "A simple fibre Fabry-Perot sensor,” Optics Communications, vol. 45, no. 2, pp. 71-74, 1983.

[10] P. J. Henderson, Y. J. Rao, D. A. Jackson, L. Zhang, and I. Bennion, "Simultaneous multi-parameter monitoring using a serial fibre-Fabry-Perot array with low-coherence and wavelength-domain detection," Measurement Science and Technology, vol. 9, no. 11, p. 1837, 1998.

[11] X. Bao, J. Dhliwayo, N. Heron, D. J. Webb, and D. A. Jackson, "Experimental and theoretical studies on a distributed temperature sensor based on Brillouin scattering," Journal of Lightwave Technology, vol. 13, no. 7, pp. 1340-1348, 1995.

[12] M. A. Farahani and T. Gogolla, "Spontaneous Raman scattering in optical fibers with modulated probe light for distributed temperature Raman remote sensing," Journal of Lightwave Technology, vol. 17, no. 8, pp. 1379-1391, 1999.

[13] E. Udd, Ed., Fiber Optic Smart Structures, Wiley, New York, NY, USA, 1995.

[14] D. Balageas, C.-P. Fritzen, and A. Guemes, Eds., Structural Health Monitoring, Wiley-ISTE, London, UK, 2006.

[15] R. Maaskanta, T. Alaviea, R. Measuresa, G. Tadrosb, S. Rizkallac, and A. Guha-Thakurtad, "Fiber-optic Bragg grating sensors for bridge monitoring," Cement Concrete Composites, vol. 19, no. 1, pp. 21-33, 1997.

[16] D. Inaudi, S. Vurpillot, N. Casanova, and P. Kronenberg, "Structural monitoring by curvature analysis using interferometric fiber optic sensors," Smart Materials and Structures, vol. 7, no. 2, pp. 199-208, 1998.

[17] W. L. Schulz, E. Udd, J. M. Seim, and G. E. McGill, "Advanced fiber grating strain sensor systems for bridges, structures, and highways," in Smart Structures and Materials 1998 Smart Systems for Bridges, Structures, and Highways, vol. 3325 of Proceedings of SPIE, pp. 212-221, March 1998.

[18] L. Thévenaz, M. Facchini, A. Fellay, P. Robert, D. Inaudi, and B. Dardel, "Monitoring of large structure using distributed brillouin fibre sensing," in Proceedings of the 13th International Conference on Optical Fiber Sensor, vol. 3746, pp. 345-348, 1999.

[19] D. R. Hjelme, L. Bjerkan, S. Neegard, J. S. Rambech, and J. V. Aarsnes, "Application of Bragg grating sensors in the characterization of scaled marine vehicle models," Applied Optics, vol. 36, no. 1, pp. 328-336, 1997.

[20] P. D. Foote, "Fibre Bragg grating strain sensors for aerospace smart structures. ," in Second European Conference on Smart Structures and Materials, vol. 2361 of Proceedings of SPIE, pp. 290-293, 1994.

[21] W. Staszewski, C. Boller, and G. Tomlinson, Eds., Health Monitoring of Aerospace Structures, John Wiley \& Sons, Chichester, UK, 2004.

[22] K. Uchiyama, K. Nonaka, and H. Takara, "Subpicosecond timing control using optical double-pulses correlation measurement," IEEE Photonics Technology Letters, vol. 16, no. 2, pp. 626-628, 2004.

[23] K. Nonaka, M. Sato, and T. Suzuki, “Optical pulse timing drift sensing for fiber delay monitoring using pulse correlation and differential detection," in Proceedings of the 17th International Conference on Optical Fibre Sensors, vol. 5855 of Proceedings of SPIE, p. 76, 2005.

[24] H. B. Song, T. Suzuki, M. Sato, and K. Nonaka, "High time resolution fibre optic sensing system based on correlation and differential technique," Measurement Science and Technology, vol. 17, no. 4, p. 631, 2006.

[25] H. B. Song, T. Suzuki, T. Fujimura, K. Nonaka, T. Shioda, and T. Kurokawa, "Polarization fluctuation suppression and sensitivity enhancement of an optical correlation sensing system," Measurement Science and Technology, vol. 18, no. 10, p. 3230, 2007.

[26] X. J. Xu and K. Nonaka, "High-sensitivity fiber-optic temperature sensing system based on optical pulse correlation and time-division multiplexer technique," Japanese Journal of Applied Physics, vol. 48, Article ID 102403, 5 pages, 2009. 
[27] X. Xu, A. Bueno, K. Nonaka, and S. Sales, "Fiber strain measurement for wide region quasidistributed sensing by optical correlation sensor with region separation techniques," Journal of Sensors, vol. 2010, Article ID 839803, 10 pages, 2010.

[28] "We experimentally measured the strain sensitivity by stretching the optical fiber". 

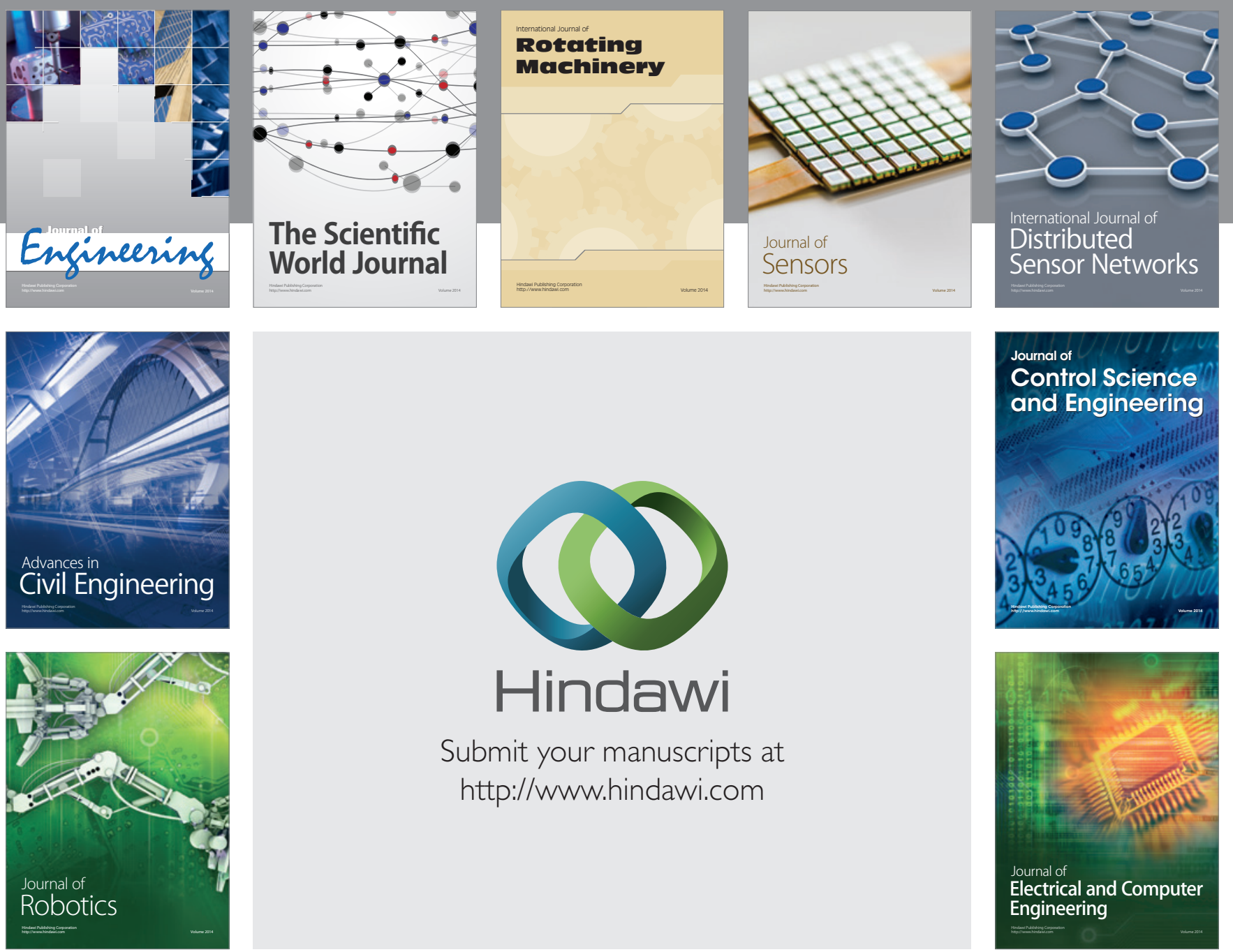

Submit your manuscripts at

http://www.hindawi.com
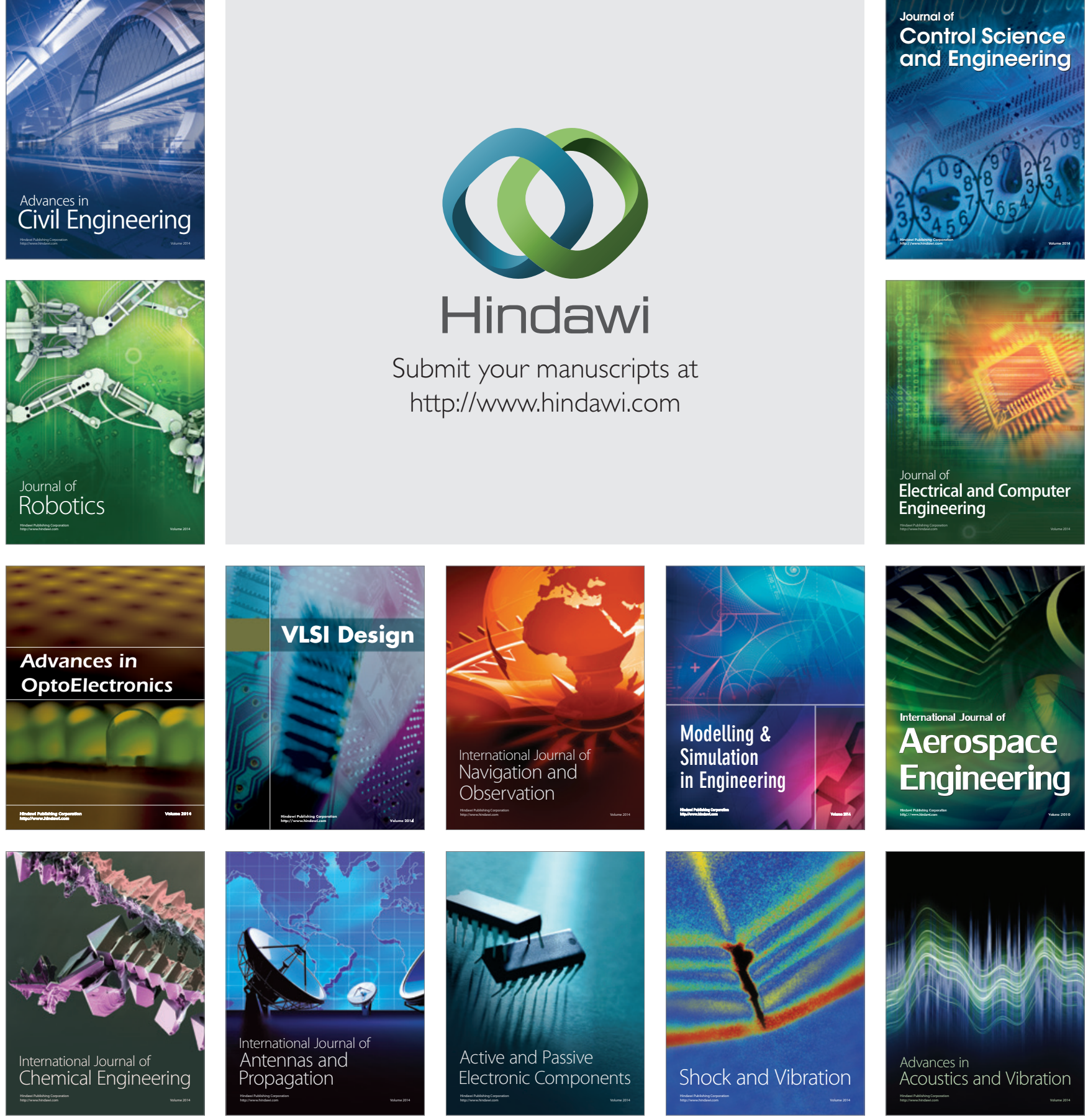